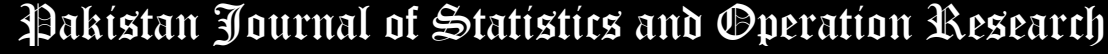

\section{The Extended Marshall-Olkin Burr III Distribution: Properties and Applications}

\author{
Muhammad Ahsan ul Haq ${ }^{1}$, Ahmed Z. Afify ${ }^{2 *}$, Hazem Al-Mofleh ${ }^{3}$, Rana \\ Muhammad Usman ${ }^{1}$, Mohammed Alqawba ${ }^{4}$, Abdullah M. Sarg ${ }^{5}$ \\ * Corresponding Author
}

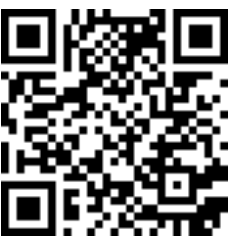

1. College of Statistical \& Actuarial Sciences, University of the Punjab, Pakistan, ahsanshani36@gmail.com, usmanrana0331@gmail.com

2. Department of Statistics, Mathematics and Insurance, Benha University, Egypt, ahmed.afify@fcom.bu.edu.eg

3. Department of Mathematics, Tafila Technical University, Tafila 66110, Jordan, almof1hm@cmich.edu

4. Department of Mathematics, College of Science and Arts, Qassim University, Ar Rass, Saudi Arabia;

m.alqawba@qu.edu.sa

5. Department of Statistics, Mathematics and Insurance, Benha University, Egypt, abdabdosasa@gmail.com

\begin{abstract}
We study a new continuous distribution called the Marshall-Olkin modified Burr III distribution. The density function of the proposed model can be expressed as a mixture of modified Burr III densities. A comprehensive account of its mathematical properties is derived. The model parameters are estimated by the method of maximum likelihood. The usefulness of the derived model is illustrated over other distributions using a real data set.
\end{abstract}

Key Words: Lifetime data; Marshall-Olkin family; Maximum likelihood; Modified Burr III; Moments; Order statistic.

Mathematical Subject Classification: 60E05, 62E15.

\section{Introduction}

Recently, several lifetime models have been derived and used in modeling data in several areas. In 1942, Burr proposed a system of twelve types of distribution functions based on generating the Pearson differential equation (Burr, 1942). Among these Burr distributions, the Burr III model which is extensively used to model data in several fields. For example, forestry data (Gove et al., 2008 and Lindsay et al., 1996), fracture roughness data (Nadarajah and Kotz, 2006a and 2007), life testing (Wingo, 1993), meteorology (Mielke, 1973), modeling crop rice (Tejeda and Goodwin, 2008) and reliability data (Abdel-Ghaly et al., 1997).

Furthermore, AL-Huniti and AL-Dayian (2012) developed a discrete version of the Burr III model, Ali et al. (2015) proposed modified Burr III (MBIII) distribution, Ali and Ahmad (2015) defined the transmuted modified Burr III distribution, and Haq et al. (2020a) proposed the unit-modifed Burr III distribution.

The probability density function (pdf) of the MBIII distribution is defined (for $x>0$ ) by

$$
g(x)=\alpha \beta x^{-\beta-1}\left(1+\gamma x^{-\beta}\right)^{-\frac{\alpha}{\gamma}-1}
$$

where $\gamma>0$ is a scale parameter and $\alpha>0$ and $\beta>0$ are shape parameters.

The cumulative distribution function (cdf) and hazard rate function (hrf) of the MBIII distribution are given by

$$
G(x)=\left(1+\gamma x^{-\beta}\right)^{-\frac{\alpha}{\gamma}}
$$

and 


$$
h(x)=\frac{\alpha \beta x^{-\beta-1}\left(1+\gamma x^{-\beta}\right)^{-\frac{\alpha}{\gamma}-1}}{1-\left(1+\gamma x^{-\beta}\right)^{-\frac{\alpha}{\gamma}}} .
$$

In this paper, we propose and study a new four-parameter model called the Marshall-Olkin modified Burr III (MOMBIII) distribution. In fact, we constrcut the new model based on the Marshall-Olkin-G (MO-G) family proposed by Marshall and Olkin (1997). Further, we provide an account of its mathematical properties.

The MO-G family (Marshall and Olkin, 1997) has been used extensively to generalize many well-known distributions. For example: the MO exponential and MO Weibull due to Marshall and Olkin (1997), MO Pareto due to Alice and Jose (2003), MO gamma due to Ristic et al. (2007), MO Lomax due to Ghitany et al. (2007), MO Lindley due to Ghitany et al. (2012), MO Fréchet due to Krishna et al. (2013), MO Birnbaum-Saunders due to Lemonte (2013), MO extended generalized Rayleigh due to MirMostafaee et al. (2017), MO exponentiated Burr XII due to Cordeiro et al. (2017), MO additive Weibull due to Afify et al. (2018), MO length biased exponential due to Haq et al. (2019), MO generalized Burr XII generalized Burr XII due to Afify and Abdellatif (2020), MO power Lomax by Haq et al. (2020b), MO inverted Nadarajah-Haghighi by Raffiq et al. (2020), MO inverted Kumaraswamy by Usman et al. (2020), and MO power generalized Weibull due to Afify et al. (2020) distributions, among others. Furthermore, the Marshall Olkin alpha power, Marshall Olkin Burr III, and Marshall-Olkin Burr-R families were proposed by Nassar et al. (2019), Afify et al. (2020) and Al-Babtain et al. (2021). Furtehr, there are some recent one parameter models which can be extended by the MO transformation to increase their flexibility such as the Shanker and Ramos-Louzada distributions due to Shanker (2015) and Ramos and Louzada (2019), respectively.

Consider the baseline cdf, $G(x)$, then the survival function (sf) of MO-G family is

$$
\bar{F}(x)=\frac{\lambda \bar{G}(x)}{[1-\bar{\lambda} \bar{G}(x)]}
$$

where $\bar{G}(x)=1-G(x)$ is the baseline sf and $\bar{\lambda}=1-\lambda, \lambda>0$ is a shape parameter. For $\lambda=1$, we obtain the baseline distribution.

The pdf of MO-G family reduces to

$$
f(x)=\frac{\lambda g(x)}{[1-\bar{\lambda} \bar{G}(x)]^{2}}
$$

The hrf is given by

$$
h(x)=\frac{g(x)}{\bar{G}(x)[1-\bar{\lambda} \bar{G}(x)]} .
$$

The rest of the paper is outlined as follows: In Section 2, we define the MOMBIII distribution and provide some plots for its pdf and hrf. Some mathematical properties including linear representation for its pdf, ordinary moments, order statistics, Rényi entropy and probability weighted moments (PWMs) are calculated in Section 3. We discuss the maximum likelihood estimation of the model parameters in Section 4. In Section 5, we assess the performance of the maximum likelihood estimates via a simulation study. Using a real data set, we show the importance of the new model in Section 6. Finally, some concluding remarks are given in Section 7.

\section{The MOMBIII distribution}

The cdf of the MOMBIII distribution is given (for $x>0$ ) by

$$
F(x)=\frac{\left(1+\gamma x^{-\beta}\right)^{-\frac{\alpha}{\gamma}}}{\lambda+(1-\lambda)\left(1+\gamma x^{-\beta}\right)^{-\frac{\alpha}{\gamma}}} .
$$

The corresponding pdf comes out as 


$$
f(x)=\frac{\alpha \beta \lambda x^{-\beta-1}\left(1+\gamma x^{-\beta}\right)^{-\frac{\alpha}{\gamma}-1}}{\left[\lambda+(1-\lambda)\left(1+\gamma x^{-\beta}\right)^{-\frac{\alpha}{\gamma}}\right]^{2}}
$$

where $\alpha, \beta$ and $\lambda$ are positive shape parameters, and $\gamma$ is a positive scale parameter.

The hrf of the MOMBIII reduces to

$$
h(x)=\frac{\alpha \beta x^{-\beta-1}\left(1+\gamma x^{-\beta}\right)^{-\frac{\alpha}{\gamma}-1}}{\left[1-\left(1+\gamma x^{-\beta}\right)^{-\frac{\alpha}{\gamma}}\right]\left[\lambda+(1-\lambda)\left(1+\gamma x^{-\beta}\right)^{-\frac{\alpha}{\gamma}}\right]} .
$$

The quantile function of the MOMBIII is given by

$$
Q(u)=\left\{\gamma /\left[\left(\frac{1-(1-\lambda) u}{u-(1-\lambda) u}\right)^{\frac{\gamma}{\alpha}}-1\right]\right\}^{\frac{1}{\beta}}, 0<u<1 .
$$

Figure 1 displays some shapes of the pdf in (6) for some selected parameter values. The figure shows that the shape of the density function is flexiable from reversed J-shape to concave down shape for certain parameter values. The plots of the MOMBIII hrf are displayed in Figure 2. The MOMBIII allows for great flexibility and hence it can be very useful in many practical situations for modeling positive data. Properties of the MOMBIII distribution.

\section{Useful expansion}

In this section, we provide a useful linear representation for the pdf of the MOMBIII distribution. The pdf (6) can be expressed as

$$
f(x)=\frac{\alpha \beta x^{-\beta-1}\left(1+\gamma x^{-\beta}\right)^{-\frac{\alpha}{\gamma}-1}}{\lambda\left[1-\left(1-\lambda^{-1}\right)\left(1+\gamma x^{-\beta}\right)^{-\frac{\alpha}{\gamma}}\right]^{2}} .
$$

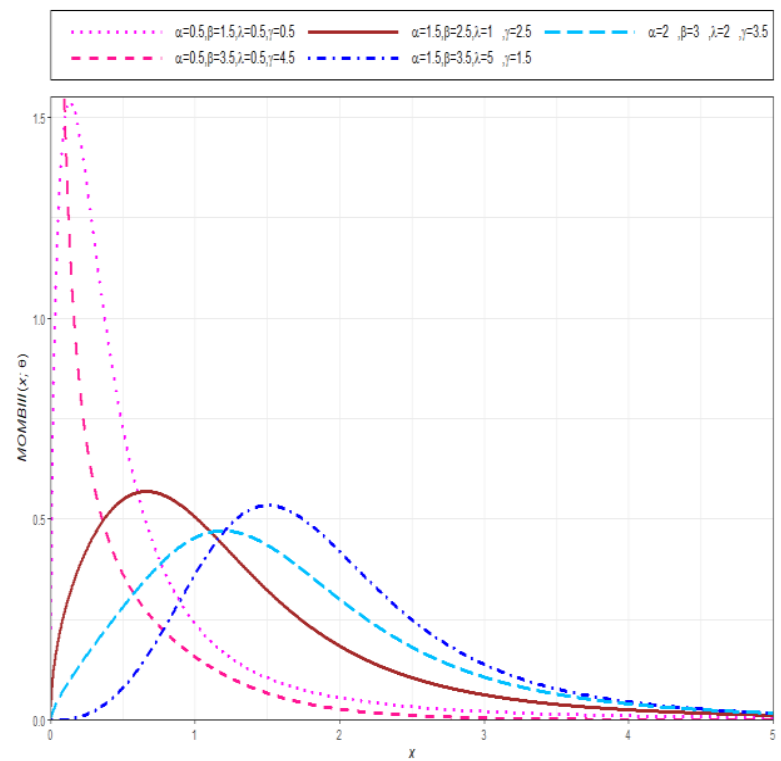

(a)

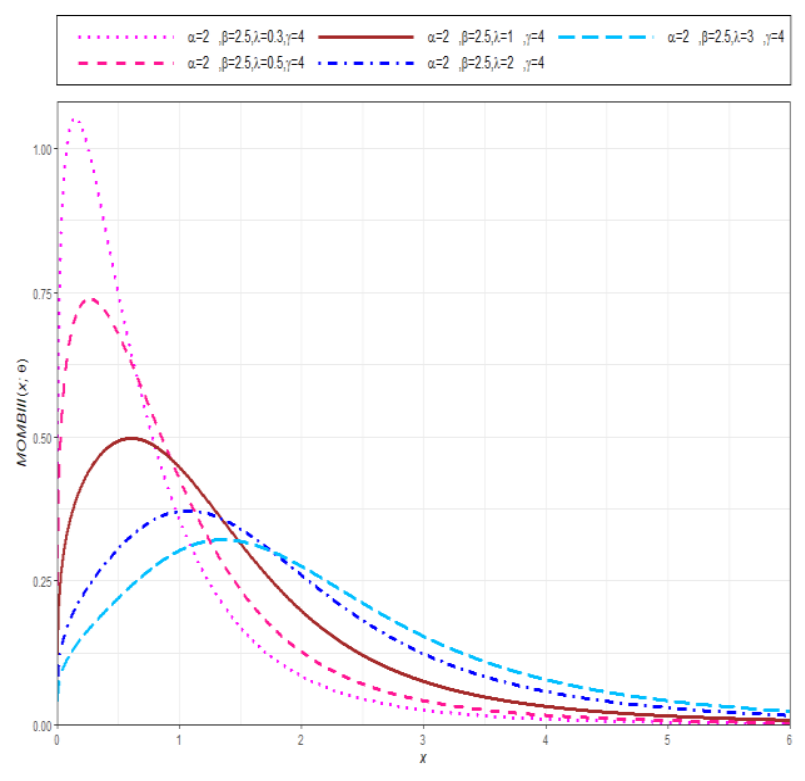

(b)

Figure 1: Plots of the MOMBIII distribution for some selected parameter values. 


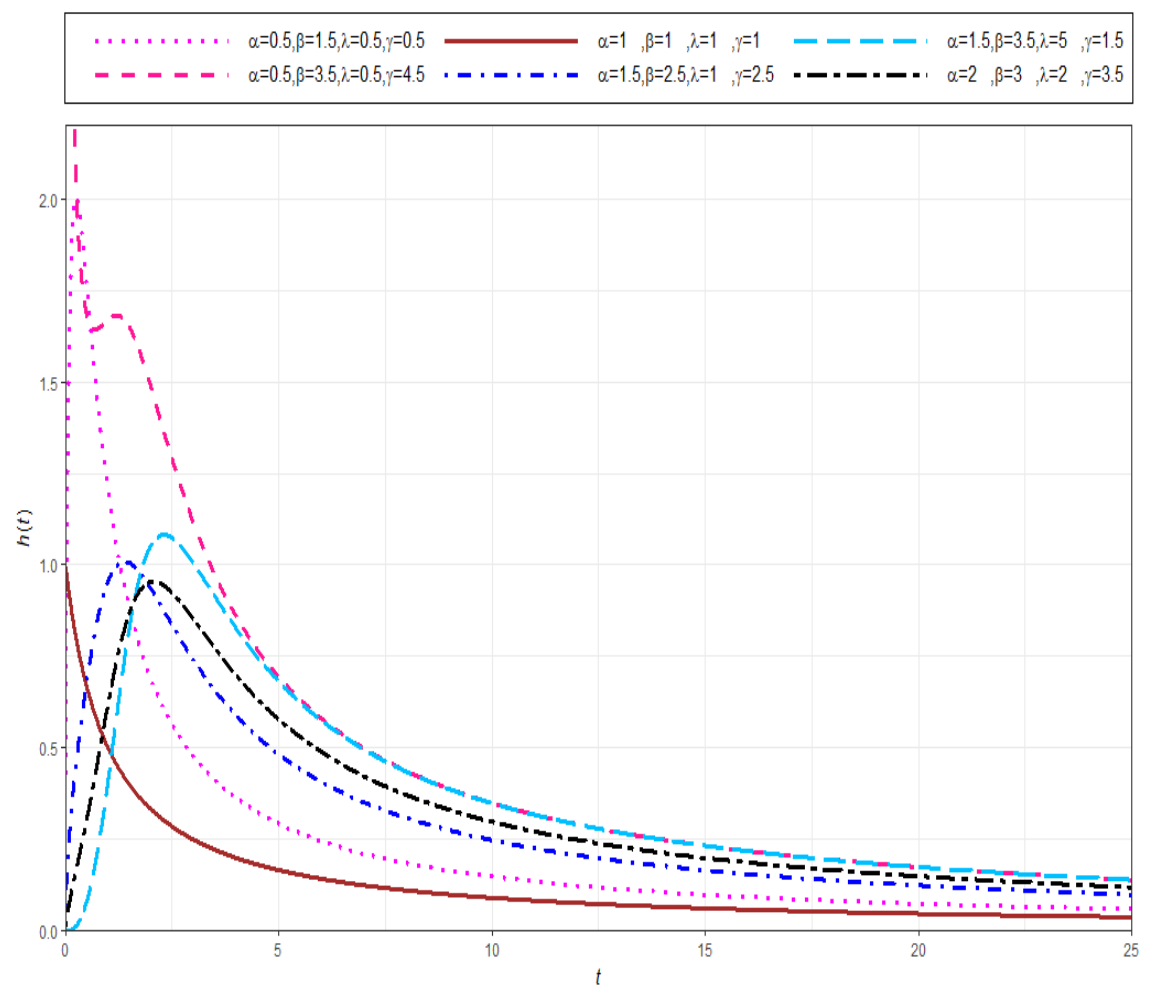

Figure 2: Plots of the MOMBIII hazard rate function (hrf) for some parameter values.

For $|a|<1$, the general binomial series holds

$$
(1-a)^{-p}=\sum_{k=0}^{\infty} \frac{\Gamma(p+k)}{k ! \Gamma(p)} a^{k}, p>0
$$

Using (8) in (7), we have

$$
f(x)=\sum_{k=0}^{\infty} \frac{\left(1-\lambda^{-1}\right)^{k}}{\lambda}(k+1) \alpha \beta x^{-\beta-1}\left(1+\gamma x^{-\beta}\right)^{-\frac{\alpha(k+1)}{\gamma}-1} .
$$

The last equation can be rewritten as

$$
f(x)=\sum_{k=0}^{\infty} w_{k} g_{(k+1) \alpha}(x) .
$$

where $w_{k}=\left(1-\lambda^{-1}\right)^{k} / \lambda$ and $g_{(k+1) \alpha}(x)$ is the density function of the MBIII with shape parameters $(k+1) \alpha$ and $\beta$ and a scale parameter $\gamma$. Based on Equation (9), we can derive the properties of the MOMBIII distribution from those of the MBIII distribution.

\section{Moments}

Let $Y$ be a random variable having the MBIII distribution, then the $r$ th ordinary moments of $Y$ is given (for $r<\beta$ ) by

$$
\mu_{r, Y}=\alpha \gamma^{\frac{r}{\beta}-1} B\left(1-\frac{r}{\beta}, \frac{r}{\beta}+\frac{\alpha}{\gamma}\right)
$$


where $B(a, b)=\int_{0}^{\infty} w^{a-1}(1+w)^{-a-b} d w$ is the beta function of the second kind. Further information abiut the MBIII distribution can be found in Ali et al. (2015).

The $r$ th moment of $X$ follows from (9) (for $r<\beta$ ) as

$$
\mu_{r}=(k+1) \alpha \gamma^{\frac{r}{\beta}-1} \sum_{k=0}^{\infty} w_{k} B\left(1-\frac{r}{\beta}, \frac{r}{\beta}+\frac{(k+1) \alpha}{\gamma}\right) .
$$

The moment generating function (mgf) of the MOMBIII distribution is given by

$$
M_{X}(t)=(k+1) \alpha \sum_{r, k=0}^{\infty} w_{k} \frac{t^{r}}{r !} \gamma^{\frac{r}{\beta}-1} B\left(1-\frac{r}{\beta}, \frac{r}{\beta}+\frac{(k+1) \alpha}{\gamma}\right) .
$$

Figure 3 shows the plots of mean and variance of the MOMBII distribution in terms of $\alpha$ and $\lambda$ when $\beta=5$ and $\gamma=$ 4. From Figure 3 and the corresponding data values (not included), the mean and the variance are increase when $\alpha$ and $\lambda$ are increse in terms of $\alpha$ and $\lambda$ when $\beta=5$ and $\gamma=4$. Figure 4 displays the plots of skewness and kurtosis of the MOMBIII distribution in terms of $\alpha$ and $\lambda$ when $\beta=5$ and $\gamma=4$. From Figure 4 and the corresponding data values (not included), the skewness is always positive which indicates that the MOMBIII distribution is right skewed, and the kurtosis is increasing function for $\alpha$ and $\lambda$.

\section{Order statistics}

Let $X_{1}, X_{2}, \ldots \ldots, X_{n}$ be a random samples of size $n$ from the MOMBIII distribution and its ordered values $\operatorname{are} X_{(1)}, X_{(2)}, \ldots \ldots, X_{(n)}$. Then, the pdf of the $i$ th order statistic, say $X_{i: n}$, can be written as

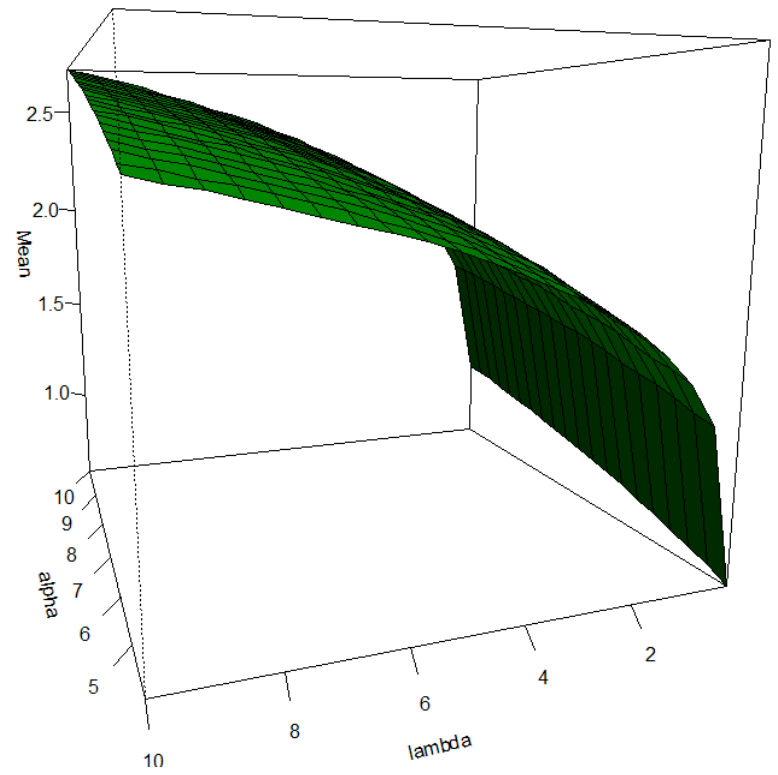

(a)

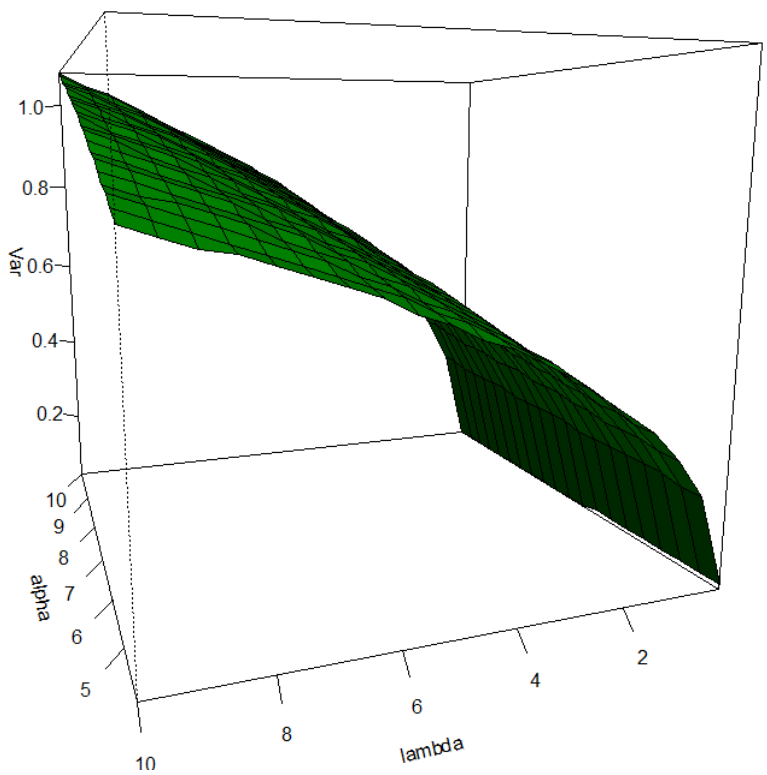

(b)

Figure 3: Plots of mean (a) and variance (b) of the MOMBIII distribution. 


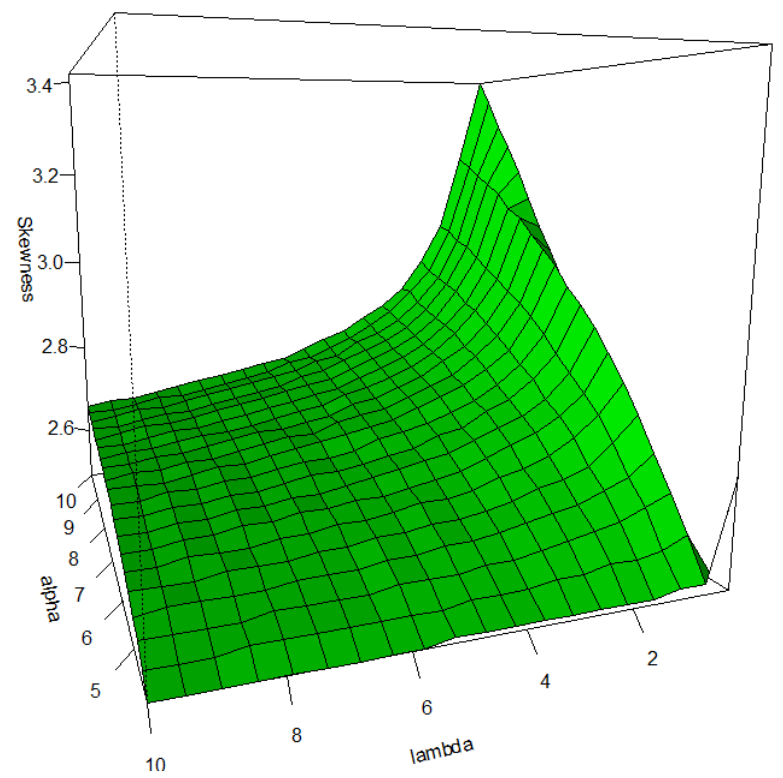

(a)

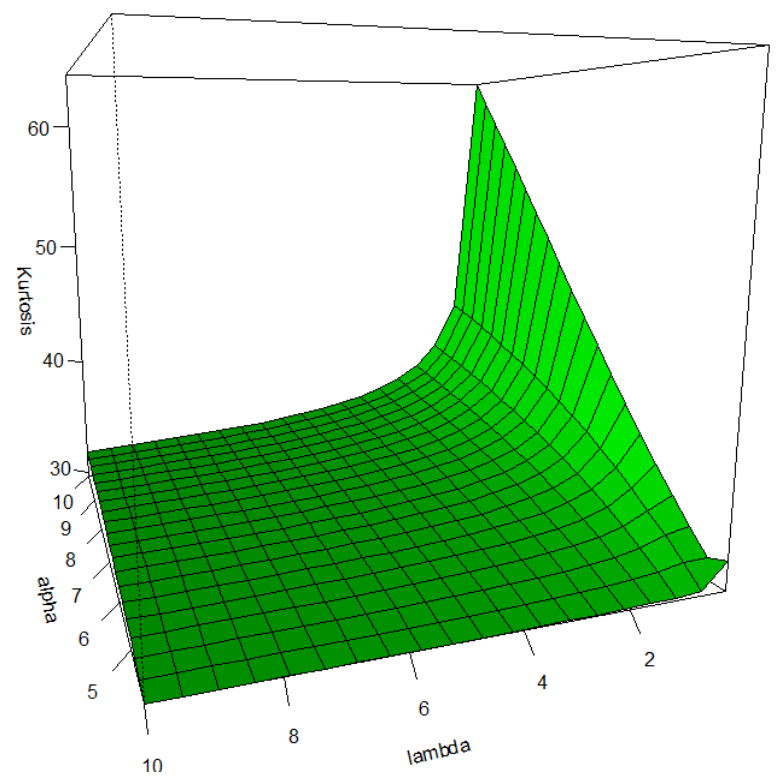

(b)

Figure 4: Plots of skewness (a) and kurtosis $(b)$ of the MOMBIII distribution.

$$
f_{i: n}(x)=\frac{n !}{(i-1) !(n-i) !} f(x) \sum_{j=0}^{n-1}(-1)^{i}\left(\begin{array}{c}
n-i \\
j
\end{array}\right) F(x)^{j+i-1} .
$$

Using (5) and (6), we have

$$
f(x) F(x)^{j+i-1}=\frac{\alpha \beta \lambda x^{-\beta-1}\left(1+\gamma x^{-\beta}\right)^{-\frac{(j+i) \alpha}{\gamma}-1}}{\left[\lambda-(\lambda-1)\left(1+\gamma x^{-\beta}\right)^{-\frac{\alpha}{\gamma}}\right]^{j+i+1}},
$$

Applying the generalized binomial series to the dominator, we obtain

$$
\left[\lambda-(\lambda-1)\left(1+\gamma x^{-\beta}\right)^{-\frac{\alpha}{\gamma}}\right]^{-(j+i+1)}=\sum_{l=0}^{\infty} \frac{(-1)^{l}}{\lambda^{j+i-1}}\left(\begin{array}{c}
-j-i-1 \\
l
\end{array}\right)\left(1-\lambda^{-1}\right)^{l}\left(1+\gamma x^{-\beta}\right)^{-\frac{l \alpha}{\gamma}}
$$

Substituting in (12), we can write

$$
f(x) F(x)^{j+i-1}=\alpha \beta x^{-\beta-1} \sum_{l=0}^{\infty} \frac{(-1)^{l}}{\lambda^{j+i-2}}\left(\begin{array}{c}
-j-i-1 \\
l
\end{array}\right)\left(1-\lambda^{-1}\right)^{l}\left(1+\gamma x^{-\beta}\right)^{-\frac{(l+j+i) \alpha}{\gamma}-1} .
$$

Combining the last equation with equation (11), we have

$$
f_{i: n}(x)=\sum_{l=0}^{\infty} \sum_{j=0}^{n-i} w_{l, j} g_{(l+j+i) \alpha}(x)
$$

where $w_{l, j}=\frac{n !(-1)^{j+l}}{(i-1) !(n-i) !} \frac{\left(1-\lambda^{-1}\right)^{l}}{\lambda^{j+i-2}(l+j+i)}\left(\begin{array}{c}n-i \\ j\end{array}\right)\left(\begin{array}{c}-j-i-1 \\ l\end{array}\right)$ and $g_{(l+j+i) \alpha}(x)$ is the pdf of the MBIII with shape parameters $(l+j+i) \alpha$ and $\beta$ and a scale parameter $\gamma$. Based on (13), we can derive some properties of the MOMBIII order statistics from those of the MBIII distribution. For example, the $s$ th moment of $X_{i: n}$ is given by 


$$
E\left(X_{i: n}^{s}\right)=\sum_{l=0}^{\infty} \sum_{j=0}^{n-i} w_{l, j}(l+j+i) \alpha \gamma^{\frac{s}{\beta}-1} B\left(1-\frac{s}{\beta}, \frac{s}{\beta}+\frac{(l+j+i) \alpha}{\gamma}\right) .
$$

\section{Rényi entropy}

The Rényi entropy of the $\operatorname{rv} X$ is a measure of variation of the uncertainty and it is defined (for $\delta>0$ and $\delta \neq 1$ ) by

$$
I_{R}(\delta)=\frac{1}{1-\delta} \log [I(\delta)],
$$

where $I(\delta)=\int_{0}^{\infty} f(x)^{\delta} d x$. Using (6), we can write

$$
I(\delta)=\alpha^{\delta} \beta^{\delta} \lambda^{\delta} \int_{0}^{\infty} x^{-\delta \beta-\delta}\left(1+\gamma x^{-\beta}\right)^{-\frac{\alpha \delta}{\gamma}-\delta}\left[\lambda-(\lambda-1)\left(1+\gamma x^{-\beta}\right)^{-\frac{\alpha}{\gamma}}\right]^{-2 \delta} d x .
$$

Using the generalized binomial series and after some simplifications, we obtain

$$
I(\delta)=\alpha^{\delta} \beta^{\delta} \sum_{l=0}^{\infty} \varphi_{l} \int_{0}^{\infty} x^{-\delta \beta-\delta}\left(1+\gamma x^{-\beta}\right)^{-\frac{(l+\delta) \alpha}{\gamma}-\delta} d x,
$$

where $\varphi_{l}=\frac{(-1)^{l}}{\lambda^{\delta}}\left(\begin{array}{c}-2 \delta \\ i\end{array}\right)\left(1-\lambda^{-1}\right)^{l}$. Let $y=\gamma x^{-\beta}$, then the last equation reduces to

$$
I(\delta)=\frac{\alpha^{\delta} \beta^{\delta-1}}{\gamma^{\frac{\delta-1}{\beta}+\delta}} \sum_{l=0}^{\infty} \varphi_{l} \int_{0}^{\infty} y^{\frac{\delta-1}{\beta}+\delta-1}(1+y)^{-\frac{(l+\delta) \alpha}{\gamma}-\delta} d y .
$$

Put $y=\frac{w}{1-w}, w=\frac{y}{1+y}$, then we obtain

$$
I(\delta)=\frac{\alpha^{\delta} \beta^{\delta-1}}{\gamma^{\frac{\delta-1}{\beta}+\delta}} \sum_{l=0}^{\infty} \varphi_{l} \int_{0}^{1} w^{\frac{\delta-1}{\beta}+\delta-1}(1-w)^{\frac{(l+\delta) \alpha}{\gamma}+\frac{1-\delta}{\beta}-1} d w .
$$

Hence,

$$
I(\delta)=\frac{\alpha^{\delta} \beta^{\delta-1}}{\gamma^{\frac{\delta-1}{\beta}+\delta}} \sum_{l=0}^{\infty} \varphi_{l} B\left(\frac{\delta-1}{\beta}+\delta, \frac{(l+\delta) \alpha}{\gamma}+\frac{1-\delta}{\beta}\right) .
$$

Then, the Rényi entropy of $\mathrm{X}$ can be expressed as

$$
I_{R}(\delta)=\frac{1}{1-\delta} \log \left[\frac{\alpha^{\delta} \beta^{\delta-1}}{\gamma^{\frac{\delta-1}{\beta}+\delta}} \sum_{l=0}^{\infty} \varphi_{l} B\left(\frac{\delta-1}{\beta}+\delta, \frac{(l+\delta) \alpha}{\gamma}+\frac{1-\delta}{\beta}\right)\right] .
$$

\section{Probability weighted moments}

The $(s, r)$ th PWM of $X$ is defined by

$$
\rho_{s, r}=E\left[X^{s} F(x)^{r}\right]=\int_{-\infty}^{\infty} x^{s} F(x)^{r} f(x) d x .
$$

Using equation (12), we can write 


$$
f(x) F(x)^{r}=\frac{\alpha \beta \lambda x^{-\beta-1}\left(1+\gamma x^{-\beta}\right)^{-\frac{(r+1) \alpha}{\gamma}-1}}{\left[\lambda-(\lambda-1)\left(1+\gamma x^{-\beta}\right)^{-\frac{\alpha}{\gamma}}\right]^{r+2}} .
$$

Using the generalized binomial series, the above equation reduces to

$$
f(x) F(x)^{r}=\alpha \beta x^{-\beta-1} \sum_{l=0}^{\infty} \frac{(-1)^{l}}{\lambda^{r-1}}\left(\begin{array}{c}
-r-2 \\
l
\end{array}\right)\left(1-\lambda^{-1}\right)^{l}\left(1+\gamma x^{-\beta}\right)^{-\frac{(l+r+1) \alpha}{\gamma}-1} .
$$

Or equivalently, we have

$$
f(x) F(x)^{r}=\sum_{l=0}^{\infty} d_{l} g_{(l+r+1) \alpha}(x),
$$

where $d_{l}=\frac{(-1)^{l}}{(l+r+1) \lambda^{r-1}}\left(\begin{array}{c}-r-2 \\ l\end{array}\right)\left(1-\lambda^{-1}\right)^{l}$ and $g_{(l+r+1) \alpha}(x)$ is the pdf of the MBIII with shape parameters $(l+r+1) \alpha$ and $\beta$ and a scale parameter $\gamma$.

Then, $\rho_{s, r}$ can be rewritten as

$$
\rho_{s, r}=\sum_{l=0}^{\infty} d_{l} \int_{0}^{\infty} x^{s} g_{(l+r+1) \alpha}(x) d x .
$$

Hence, the $(s, r)$ th PWM of $X$ comes out as

$$
\rho_{s, r}=\sum_{l=0}^{\infty} d_{l}(l+r+1) \alpha \gamma^{\frac{s}{\beta}-1} B\left(1-\frac{s}{\beta}, \frac{s}{\beta}+\frac{(l+r+1) \alpha}{\gamma}\right) .
$$

\section{Maximum likelihood estimation}

In this section, the maximum likelihood estimators (MLEs) of the MOMBIII parameters are obtained. Let $X_{1}, \cdots, X_{n}$ be a random sample of size $n$ from this distribution with parameters $\alpha, \beta, \lambda$ and $\gamma$. Let $\theta=(\alpha, \beta, \lambda, \gamma)^{\text {ú }}$ be the $p \times 1$ parameter vector. The log-likelihood function for $\theta$ reduces to

$$
\begin{aligned}
& \ell(\theta)=n \log \alpha+n \log \beta+n \log \lambda-\left(\frac{\alpha}{\gamma}+1\right) \sum_{i=1}^{n} \log \left(1+\gamma x_{i}^{-\beta}\right)-(\beta+1) \sum_{i=1}^{n} \log x_{i} \\
& \quad-2 \sum_{i=1}^{n} \log \left[\lambda+(1-\lambda)\left(1+\gamma x_{i}^{-\beta}\right)^{-\frac{\alpha}{\gamma}}\right] .
\end{aligned}
$$

The above log-likelihood can be maximized numerically using the R (optim function), SAS (PROC NLMIXED) or Ox program (sub-routine MaxBFGS)), among others.

The score vector elements are given respectively by

$$
\begin{aligned}
& \frac{\partial \ell}{\partial \alpha}=\frac{n}{\alpha}-\frac{1}{\gamma} \sum_{i=1}^{n} \log \left(1+\gamma x_{i}^{-\beta}\right)+\frac{2(1-\lambda)}{\gamma} \sum_{i=1}^{n} \frac{\left(1+\gamma x_{i}^{-\beta}\right)^{-\frac{\alpha}{\gamma}} \log \left(1+\gamma x_{i}^{-\beta}\right)}{\lambda+(1-\lambda)\left(1+\gamma x_{i}^{-\beta}\right)^{-\frac{\alpha}{\gamma}}} \\
& \frac{\partial \ell}{\partial \beta}=\frac{n}{\beta}-\sum_{i=1}^{n} \log x_{i}+(\alpha+\gamma) \sum_{i=1}^{n} \frac{x_{i}^{-\beta} \log x_{i}}{\left(1+\gamma x_{i}^{-\beta}\right)}-2 \alpha(1-\lambda) \sum_{i=1}^{n} \frac{x_{i}^{-\beta}\left(1+\gamma x_{i}^{-\beta}\right)^{-\frac{\alpha}{\gamma}-1} \log x_{i}}{\lambda+(1-\lambda)\left(1+\gamma x_{i}^{-\beta}\right)^{-\frac{\alpha}{\gamma}}}
\end{aligned}
$$




$$
\frac{\partial \ell}{\partial \lambda}=\frac{n}{\lambda}-2 \sum_{i=1}^{n} \frac{1-\left(1+\gamma x_{i}^{-\beta}\right)^{-\frac{\alpha}{\gamma}}}{\lambda+(1-\lambda)\left(1+\gamma x_{i}^{-\beta}\right)^{-\frac{\alpha}{\gamma}}}
$$

and

$$
\begin{aligned}
\frac{\partial \ell}{\partial \gamma}=\frac{\alpha}{\gamma^{2}} \sum_{i=1}^{n} \log ( & \left.+\gamma x_{i}^{-\beta}\right)-\left(\frac{\alpha}{\gamma}+1\right) \sum_{i=1}^{n} \frac{x_{i}^{-\beta}}{1+\gamma x_{i}^{-\beta}}-\frac{2 \alpha(1-\lambda)}{\gamma^{2}} \sum_{i=1}^{n} \frac{\left(1+\gamma x_{i}^{-\beta}\right)^{-\frac{\alpha}{\gamma}} \log \left(1+\gamma x_{i}^{-\beta}\right)}{\lambda+(1-\lambda)\left(1+\gamma x_{i}^{-\beta}\right)^{-\frac{\alpha}{\gamma}}} \\
+ & \frac{2 \alpha(1-\lambda)}{\gamma} \sum_{i=1}^{n} \frac{\left(1+\gamma x_{i}^{-\beta}\right)^{-\frac{\alpha}{\gamma}-1} x^{-\beta}}{\lambda+(1-\lambda)\left(1+\gamma x_{i}^{-\beta}\right)^{-\frac{\alpha}{\gamma}}}
\end{aligned}
$$

The exact solution of above derived MLEs for the unknown parameters is not possible. So it is more convenient to use nonlinear optimization algorithms such as Newton Raphson algorithm to numerically maximize the above likelihood function. For interval estimation of the parameters, we obtain the $p \times p$ observed information matrix $J(\theta)=$ $\left\{\frac{\partial^{2} \ell}{\partial r \partial s}\right\}$ (for $r, s=\alpha, \beta, \lambda, \gamma$ ), whose elements can be obtained upon request.

\section{Simulation study}

In this section, we assess the finite sample behaviors of the MLEs for the MOMBIII distribution via a Monte Carlos simulation study. We generate 10,000 samples of sizes $n=50$ and $n=300$ for different combinations of parameters. The MATHEMATICA 10.0 is used to obtain the mean values, bias and mean square errors (MSE). Table 1 lists the mean values of the estimates, bias and MSE.

One can see, from Table 1, that the estimates of model parameters are closer to true values as sample size increases and the biases and MSE decreases as $n \rightarrow \infty$.

\section{Data analysis}

In this section, we illustrate the importance and flexibility of the MOMBIII distribution using a real data set. we compare the fits of the MOMBIII model with the MBIII, beta exponential (BE) (Nadarajah and Kotz, 2006b), exponentiated modified Burr III (EMBIII), transmuted modified Burr III (TMBIII) (Ali and Ahmad, 2015), transmuted Gompertz (TG) (Abdul-Moniem and Seham, 2015) and Burr III (BIII) distributions whose pdfs are given respectively (for $x>0$ ) by

$$
\begin{aligned}
& \text { BE: } f(x ; \alpha, \beta, \lambda)=\frac{\lambda}{B(a, b)} \exp (-b \lambda x)[1-\exp (-\lambda x)]^{a-1} ; \\
& \text { EMBIII: } f(x ; \alpha, \beta, \lambda, \gamma)=\alpha \beta \lambda x^{-(\beta+1)}\left(1+\frac{\gamma}{x^{\beta}}\right)^{-\frac{\alpha \lambda}{\gamma}-1} ; \\
& \text { TMBIII: } f(x ; \alpha, \beta, \lambda, \gamma)=\alpha \beta x^{-(\beta+1)}\left(1+\frac{\gamma}{x^{\beta}}\right)^{-\frac{\alpha}{\gamma}-1}\left[1+\lambda-2 \lambda\left(1+\frac{\gamma}{x^{\beta}}\right)^{-\frac{\alpha}{\gamma}}\right] \text {; } \\
& \text { TG: } f(x ; \alpha, \beta, \lambda)=\alpha \lambda e^{\alpha x} e^{-\lambda\left(e^{\alpha x}-1\right)}\left[1-\lambda+2 \lambda e^{-\lambda\left(e^{\alpha x}-1\right)}\right] ; \\
& \text { BIII: } f(x ; \alpha, \beta)=\alpha \beta x^{-\beta-1}\left(1+x^{-\beta}\right)^{-\alpha-1} ;
\end{aligned}
$$

where the pdf of the MBIII model is given in Section 1 and all the parameters are real numbers except for the TMBIII and TG distributions for them $|\lambda| \leq 1$.

We consider a data set obtained from Smith and Naylor (1987) which represents the strengths of $1.5 \mathrm{~cm}$ glass fibers, measured at the National Physical Laboratory, England. The observations are: 0.0251, 0.0886, 0.0891, 0.2501, 0.3113, 0.3451, 0.4763, 0.5650, 0.5671, 0.6566, 0.6748, 0.6751, 0.6753, 0.7696, 0.8375, 0.8391, 0.8425, 0.8645, 0.8851, $0.9113,0.9120,0.9836,1.0483,1.0596,1.0773,1.1733,1.2570,1.2766,1.2985,1.3211,1.3503,1.3551,1.4595$, $1.4880,1.5728,1.5733,1.7083,1.7263,1.7460,1.7630,1.7746,1.8275,1.8375,1.8503,1.8808,1.8878,1.8881$, $1.9316,1.9558,2.0048,2.0408,2.0903,2.1093,2.1330,2.2100,2.2460,2.2878,2.3203,2.3470,2.3513,2.4951$, 2.5260, 2.9911, 3.0256, 3.2678, 3.4045, 3.4846, 3.7433, 3.7455, 3.9143, 4.8073, 5.4005, 5.4435, 5.5295, 6.5541, 9.0960. The descriptive summary of the data set is given in Table 2. These data were analyzed by Afify et al. (2018), Mansour et al. (2018), and Mead et al. (2019). 
Table 1: Mean estimates, bias and MSE of the MOMBIII model.

\begin{tabular}{|c|c|c|c|c|}
\hline Parameters & Sample size & Mean & Bias & MSE \\
\hline & & 1.52468 & 0.02467 & 0.03938 \\
\hline & & 0.51879 & 0.01879 & 0.01025 \\
\hline$\alpha=1.5$ & 50 & 1.50892 & 0.00891 & 0.01548 \\
\hline$\beta=0.5$ & & 1.49479 & -0.00521 & 0.00399 \\
\hline$\lambda=1.5$ & \multirow{4}{*}{300} & 1.50494 & 0.00494 & 0.00630 \\
\hline \multirow[t]{3}{*}{$\gamma=1.5$} & & 0.50255 & 0.00255 & 0.00144 \\
\hline & & 1.50222 & 0.00221 & 0.00254 \\
\hline & & 1.49907 & -0.00092 & 0.00067 \\
\hline \multirow{8}{*}{$\begin{array}{l}\alpha=1.5 \\
\beta=0.5 \\
\lambda=2.0 \\
\gamma=1.5\end{array}$} & \multirow{4}{*}{50} & 1.52065 & 0.02065 & 0.03461 \\
\hline & & 0.51860 & 0.01860 & 0.01169 \\
\hline & & 2.01308 & 0.01308 & 0.02842 \\
\hline & & 1.49530 & -0.00469 & 0.00419 \\
\hline & \multirow{4}{*}{300} & 1.50350 & 0.00349 & 0.00545 \\
\hline & & 0.50288 & 0.00288 & 0.00161 \\
\hline & & 2.00255 & 0.00254 & 0.00446 \\
\hline & & 1.49937 & -0.00062 & 0.00072 \\
\hline \multirow{8}{*}{$\begin{array}{l}\alpha=1.5 \\
\beta=0.5 \\
\lambda=4.0 \\
\gamma=1.5\end{array}$} & \multirow{4}{*}{50} & 1.51022 & 0.01022 & 0.00833 \\
\hline & & 0.50593 & 0.00593 & 0.00201 \\
\hline & & 4.00474 & 0.00474 & 0.00939 \\
\hline & & 1.48335 & -0.01665 & 0.00928 \\
\hline & \multirow{4}{*}{300} & 1.50451 & 0.00451 & 0.00604 \\
\hline & & 0.50122 & 0.00122 & 0.00039 \\
\hline & & 4.00031 & 0.00031 & 0.00865 \\
\hline & & 1.49303 & -0.00697 & 0.00851 \\
\hline \multirow{8}{*}{$\begin{array}{l}\alpha=0.5 \\
\beta=0.5 \\
\lambda=0.5 \\
\gamma=0.5\end{array}$} & \multirow{4}{*}{50} & 0.51449 & 0.01449 & 0.00839 \\
\hline & & 0.52244 & 0.02244 & 0.01415 \\
\hline & & 0.50317 & 0.00317 & 0.00176 \\
\hline & & 0.49916 & -0.00084 & 0.00037 \\
\hline & \multirow{4}{*}{300} & 0.50143 & 0.00143 & 0.00121 \\
\hline & & 0.50238 & 0.00238 & 0.00177 \\
\hline & & 0.50021 & 0.00021 & 0.00028 \\
\hline & & 0.50007 & 0.00007 & 0.00006 \\
\hline \multirow{8}{*}{$\begin{array}{l}\alpha=0.5 \\
\beta=0.5 \\
\lambda=1.0 \\
\gamma=0.5\end{array}$} & \multirow{4}{*}{50} & 0.51005 & 0.10058 & 0.00546 \\
\hline & & 0.51616 & 0.01616 & 0.00968 \\
\hline & & 1.00656 & 0.00655 & 0.00701 \\
\hline & & 0.49872 & -0.00127 & 0.00040 \\
\hline & \multirow{4}{*}{300} & 0.50195 & 0.00195 & 0.00084 \\
\hline & & 0.50256 & 0.00256 & 0.00136 \\
\hline & & 1.00145 & 0.00144 & 0.00114 \\
\hline & & 0.49972 & -0.00027 & 0.00006 \\
\hline \multirow{8}{*}{$\begin{array}{l}\alpha=0.5 \\
\beta=0.5 \\
\lambda=5.0 \\
\gamma=0.5\end{array}$} & \multirow{4}{*}{50} & 0.50471 & 0.00471 & 0.00266 \\
\hline & & 0.51880 & 0.01880 & 0.01056 \\
\hline & & 5.03069 & 0.03069 & 0.17321 \\
\hline & & 0.49742 & -0.00257 & 0.00059 \\
\hline & \multirow{4}{*}{300} & 0.50009 & 0.00096 & 0.00043 \\
\hline & & 0.50313 & 0.00312 & 0.00146 \\
\hline & & 5.00584 & 0.00584 & 0.02818 \\
\hline & & 0.49943 & -0.00056 & 0.00010 \\
\hline \multirow{8}{*}{$\begin{array}{l}\alpha=1.5 \\
\beta=0.5 \\
\lambda=0.5 \\
\gamma=1.5\end{array}$} & & 1.54290 & 0.04289 & 0.07628 \\
\hline & 50 & 0.51502 & 0.01502 & 0.00879 \\
\hline & 50 & 0.50314 & 0.00314 & 0.00176 \\
\hline & & 1.49787 & -0.00213 & 0.00347 \\
\hline & & 1.50649 & 0.00649 & 0.01120 \\
\hline & & 0.50218 & 0.00218 & 0.00124 \\
\hline & 300 & 0.50052 & 0.00052 & 0.00028 \\
\hline & & 1.49981 & -0.00019 & 0.00057 \\
\hline
\end{tabular}


Table 2: Descriptive statistics for glass fibers data

\begin{tabular}{|l|l|l|l|l|l|l|l|}
\hline$n$ & Min. & Median & Max. & Mean & S.d. & Sk. & Ku. \\
\hline & 0.0251 & 1.7362 & 9.0960 & 1.9592 & 1.5740 & 1.9796 & 8.1608 \\
\hline
\end{tabular}

Table 3: Parameter estimates (standard errors of the MLE in parentheses) and goodness-of-fit statistics for glass

\begin{tabular}{|c|c|c|c|c|c|c|c|}
\hline \multicolumn{8}{|c|}{ fibers data } \\
\hline & MOMBIII & TMBIII & MBIII & EMBIII & TG & $\mathrm{BE}$ & BIII \\
\hline \multirow{11}{*}{$\begin{array}{c}M L E \\
(\mathrm{SE})\end{array}$} & $\hat{\alpha}$ & $\hat{\alpha}$ & $\hat{\alpha}$ & $\hat{\alpha}$ & $\hat{\alpha}$ & $\hat{\alpha}$ & $\hat{\alpha}$ \\
\hline & 1759.850 & 10770.537 & 38395.869 & 215219.260 & 2.937 & 17.450 & 3.442 \\
\hline & $(5613.156)$ & (3198.458) & (5352.319) & (13441.582) & $(0.336)$ & (3.081) & $(0.450)$ \\
\hline & $\hat{\beta}$ & $\hat{\beta}$ & $\hat{\beta}$ & $\hat{\beta}$ & $\hat{\beta}$ & $\hat{\beta}$ & $\hat{\beta}$ \\
\hline & 18.584 & 19.428 & 20.70 & 20.713 & 0.012 & 163.862 & 4.089 \\
\hline & (4.579) & $(0.746)$ & $(0.739)$ & $(0.740)$ & (0.008) & (252.109) & $(0.336)$ \\
\hline & $\hat{\lambda}$ & $\hat{\lambda}$ & $\hat{\lambda}$ & $\hat{\lambda}$ & $\hat{\lambda}$ & $\hat{\lambda}$ & \\
\hline & 4.842 & -0.533 & 172895.570 & 0.179 & -0.748 & 0.067 & \\
\hline & (4.893) & $(0.324)$ & (8828.504) & (0.028) & $(0.266)$ & (0.098) & \\
\hline & $\hat{\gamma}$ & $\hat{\gamma}$ & & $\hat{\gamma}$ & & & \\
\hline & $\begin{array}{c}12873.855 \\
(41047.139)\end{array}$ & $\begin{array}{l}56654.959 \\
(9385.550)\end{array}$ & - & $\begin{array}{c}173318.269 \\
(9217.809)\end{array}$ & - & - & - \\
\hline$-2 L$ & 20.248 & 22.204 & 23.826 & 23.826 & 27.894 & 47.914 & 73.767 \\
\hline AIC & 28.248 & 30.204 & 29.826 & 31.826 & 33.894 & 53.914 & 77.767 \\
\hline CAIC & 28.938 & 30.894 & 30.233 & 32.515 & 34.301 & 54.320 & 77.967 \\
\hline$B I C$ & 36.821 & 38.776 & 36.255 & 40.398 & 40.323 & 60.343 & 82.054 \\
\hline$H Q I C$ & 31.620 & 33.576 & 32.354 & 35.197 & 36.422 & 56.442 & 79.453 \\
\hline$W$ & 0.039 & 0.083 & 0.114 & 0.114 & 0.130 & 0.569 & 0.955 \\
\hline$A$ & 0.239 & 0.465 & 0.627 & 0.627 & 0.752 & 3.118 & 5.200 \\
\hline$K-S$ & 0.075 & 0.109 & 0.129 & 0.129 & 0.121 & 0.216 & 0.246 \\
\hline$p$-value & 0.874 & 0.441 & 0.243 & 0.244 & 0.3180 & 0.006 & 0.001 \\
\hline
\end{tabular}

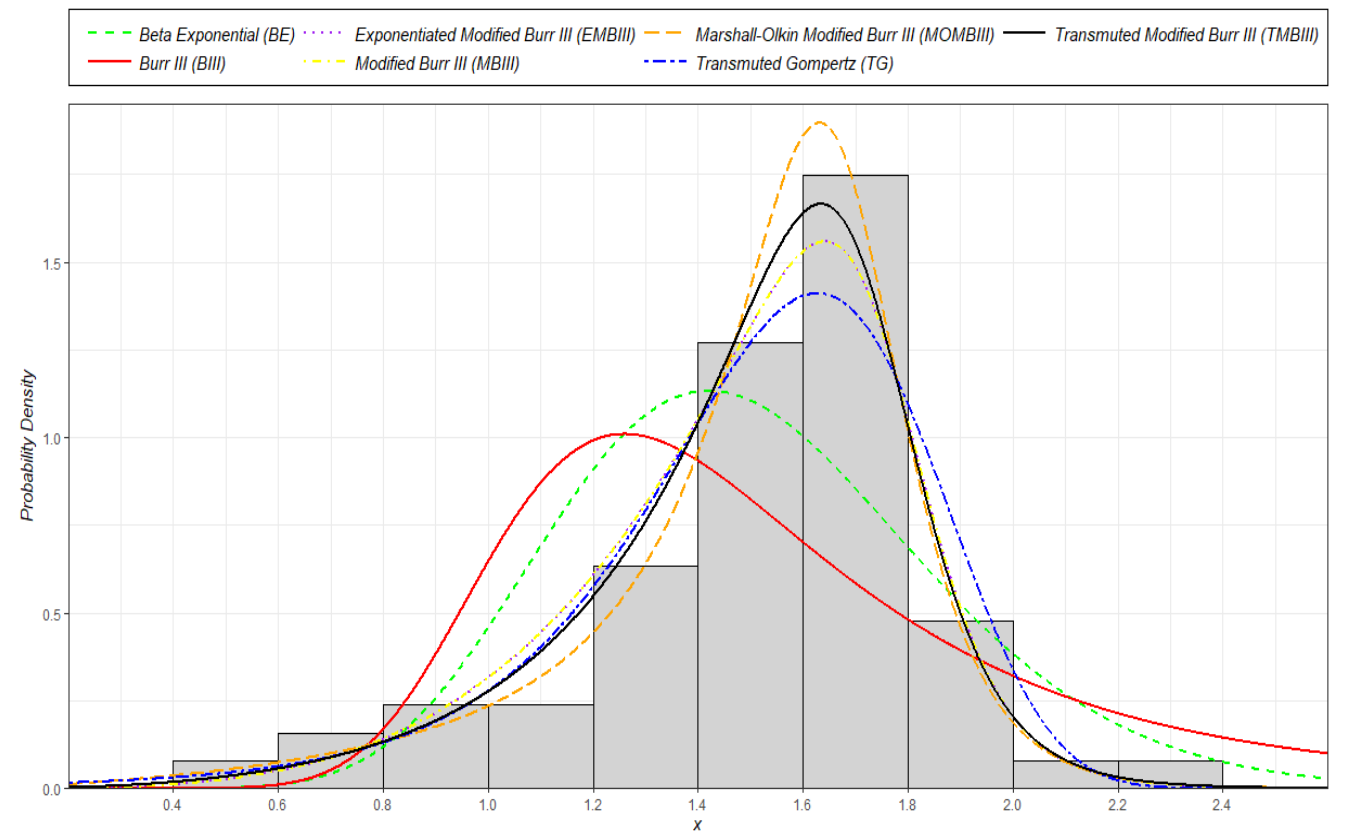

Figure 5: The fitted pdfs of the MOMBIII model and other fitted models. 
The distribution parameters are estimated by using the maximum likelihood method. The estimated values are given in Table 3. In order to compare the fitted distribution, we used the following goodness-of-fit statistics: -2 log-likelihood $(-2 L)$, Akaike information criterion $(A I C)$, corrected Akaike information criterion (CAIC), Bayesian information criterion $(B I C)$ and Hannan-Quinn Information Criterion $(H Q I C)$. Along with these measures some most commonly used statistics are also used, such as: Durbin-Watson test $W$, Anderson Darling test $A$ and Kolmogorov-Smirnov (KS) test statistic with its corresponding p-value. Table 3 shows the goodness-of-fit statistic results.

It can be concluded that the MOMBIII distribution has the lowest $-2 L, A I C, C A I C, B I C, H Q I C, W, A$ and the $\mathrm{K}-\mathrm{S}$ statistic values, and the largest p-values among all the other models, hence, the MOMBIII distribution could be chosen as the best model. The relative histogram and the fitted distributions is displayed in Figure 5. Also, the plots of the fitted cdfs and empirical cdf of the data are displayed in Figure 6.

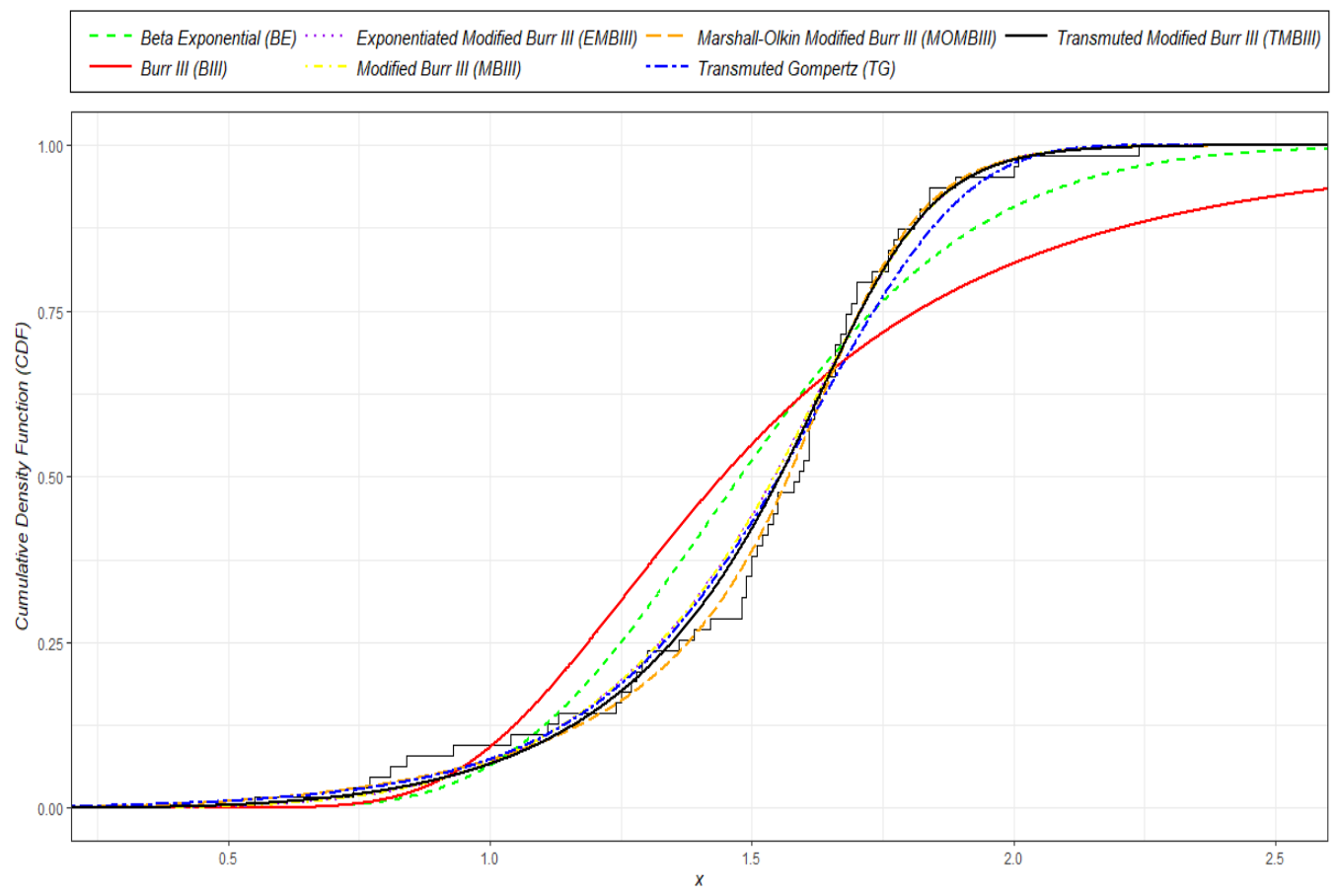

Figure 6: The estimated cdfs of the MOMBIII model and other models.

\section{Conclusion}

In this article, we propose a new four-parameter lifetime model called the Marshall-Olkin modified Burr III (MOMBIII) distribution which extends the modified Burr III (MBIII) model. The density function of the MOMBIII model can be expressed as a mixture of MBIII densities. We provide some mathematical properties of the MOMBIII distribution including explicit expressions for the ordinary moments, order statistics, Rényi entropy and probability weighted moments. The unkown parameters of the new model are estimated by maximum-likelihood and a Monte Carlo simulation study to assess the finite sample behavior of the maximum-likelihood estimators is performed. By means of an application to real data, we prove empirically that the MOMBIII distribution can give better fits than other well-known models in the literature.

\section{References}

1. Abdel-Ghaly, A., Al-Dayian, G. and Al-Kashkari, F. (1997). The use of Burr type XII distribution on software reliability growth modeling. Microelectronics Reliability, 37, 305-313.

2. Abdul-Moniem, I. and Seham, M. (2015). Transmuted Gompertz distribution. Computational and Applied Mathematics Journal, 1, 88-96.

3. Afify, A. Z. and Abdellatif, A. D. (2020). The extended Burr XII distribution: properties and applications. J. of Nonlinear Sci. and Appl, 13, 133-146. 
4. Afify, A. Z., Cordeiro, G. M., Ibrahim, N. A., Jamal, F., Elgarhy, M. and Nasir, M. A. (2020). The Marshall-Olkin odd Burr III-G family: theory, estimation, and engineering applications, IEEE Access, doi: 10.1109/ACCESS.2020.3044156.

5. Afify A. Z., Cordeiro G. M., Yousof, H. M., Saboor, A. and Ortega, E. M. M. (2018). The MarshallOlkin additive Weibull distribution with variable shapes for the hazard rate. Hacettepe Journal of Mathematics and Statistics, 47, 365-381.

6. Afify, A. Z., Kumar, D. and Elbatal, I. (2020). Marshall-Olkin power generalized Weibull distribution with applications in engineering and medicine. Journal of Statistical Theory and Applications, 19, 223237.

7. Afify, A. Z., Zayed, M. and Ahsanullah, M. (2018). The extended exponential distribution and its applications. Journal of Statistical Theory and Applications, 17, 213-229.

8. Al-Babtain, A. A., Sherwani, R. A. K., Afify, A. Z., Aidi, K., Nasir, M. A., Jamal, A. and Saboor, A. (2021). The extended Burr-R class: properties, applications and modified test for censored data. AIMS Mathematics, 6, 2912-2931.

9. Al-Huniti, A. A. and AL-Dayian, G. R. (2012). Discrete Burr type III distribution. American Journal of Mathematics and Statistics, 2, 145-152.

10. Ali, A. and Ahmad, M. (2015). The transmuted modified Burr III distribution. Journal of ISOSS, 1, 119130.

11. Ali, A., Hasnain, S. A. and Ahmad, M. (2015). Modified Burr III distribution, properties and applications. Pak. J. Statist, 31(6), 697-708.

12. Alice, T. and Jose, K. (2003). Marshall-Olkin Pareto processes. Far East Journal of Theoretical Statistics, 9(2), 117-132.

13. Burr, I. W. (1942). Cumulative frequency functions. The Annals of mathematical statistics, 13(2), 215 232.

14. Cordeiro, G., Mead, M., Afify, A. Z., Suzuki, A. and Abd El-Gaied, A. (2017). An extended Burr XII distribution: properties, inference and applications. Pakistan Journal of Statistics and Operation Research, 13, 809-828.

15. Ghitany, M., Al-Mutairi, D., Al-Awadhi, F. and Al-Burais, M. (2012). Marshall-Olkin extended Lindley distribution and its application. International Journal of Applied Mathematics, 25(5), 709-721.

16. Ghitany, M., Al-Awadhi, F. and Alkhalfan, L. (2007). Marshall-Olkin extended Lomax distribution and its application to censored data. Communications in Statistics-Theory and Methods, 36(10), 1855-1866.

17. Gove, J. H., Ducey, M. J., Leak, W. B. and Zhang, L. (2008). Rotated sigmoid structures in managed uneven-aged northern hardwood stands: a look at the Burr Type III distribution. Forestry, 81, 161-176.

18. Haq, M. A. U., Hashmi, S., Aidi, K., Ramos, P. L. and Louzada, F. (2020a). Unit modified Burr-III distribution: estimation, characterizations and validation test. Annals of Data Science. DOI: org/10.1007/s40745-020-00298-6.

19. Haq, M. A. U., Hamedani, G. G., Elgarhy, M. and Ramos, P. L. (2020b). Marshall-Olkin Power Lomax distribution: Properties and estimation based on complete and censored samples. Int. J. Stat. Probab, 9, 48-62.

20. Haq, M. A., Usman, R. M., Hashmi, S. and Al-Omeri, A. I. (2019). The Marshall-Olkin length-biased exponential distribution and its applications. Journal of King Saud University-Science. 31, $246-251$.

21. Krishna, E., Jose, K., Alice, T. and Ristic', M. M. (2013). The Marshall-Olkin Fréchet distribution. Communications in Statistics-Theory and Methods, 42, 4091-4107.

22. Lindsay, S., Wood, G. and Woollons, R. (1996). Modeling the diameter distribution of forest stands using the Burr distribution. Journal of Applied Statistics, 23(6), 609-620.

23. Mansour, M. M., Aryal, G., Afify, A. Z. and Ahmad, M. (2018). The Kumaraswamy exponentiated Fréchet distribution. Pakistan Journal of Statistics, 34, 177-193.

24. Marshall, A. W. and Olkin, I. (1997). A new method for adding a parameter to a family of distributions with application to the exponential and Weibull families. Biometrika, 84, 641-652.

25. Mead, M. E., Cordeiro, G. M., Afify, A. Z. and Al Mofleh, H. (2019). The alpha power transformation family: properties and applications. Pakistan Journal of Statistics and Operation Research, 15, 525-545.

26. Mielke Jr, P. W. (1973). Another family of distributions for describing and analyzing precipitation data. Journal of Applied Meteorology, 12, 275-280.

27. MirMostafaee, S., Mahdizadeh, M. and Lemonte, A. J. (2017). The Marshall-Olkin extended generalized Rayleigh distribution: Properties and applications. Communications in Statistics-Theory and Methods, 46, 653-671. 
28. Nadarajah, S. and Kotz, S. (2006a). q exponential is a Burr distribution. Physics Letters A, 359, 577579.

29. Nadarajah, S. and Kotz, S. (2006b). The beta exponential distribution. Reliability engineering \& system safety, 91(6), 689-697.

30. Nadarajah, S. and Kotz, S. (2007). On the alternative to the Weibull function. Engineering Fracture Mechanics, 74(3), 451-456.

31. Nassar, M., Kumar, D., Dey, S., Cordeiro, G. M. and Afify, A. Z. (2019). The Marshall-Olkin alpha power family of distributions with applications. Journal of Computational and Applied Mathematics, 351, 41-53.

32. Raffiq, G., Dar, I. S., Haq, M. A. U. and Ramos, E. (2020). The Marshall-Olkin inverted NadarajahHaghighi distribution: estimation and applications. Annals of Data Science. DOI: org/10.1007/s40745020-00297-7.

33. Ramos, P. L. and Louzada, F. (2019). A distribution for instantaneous failures. Stats, 2, 247-258.

34. Ristic, M. M., Jose, K. and Ancy, J. (2007). A Marshall-Olkin gamma distribution and minification process. STARS: Stress Anxiety Res Soc, 11, 107-117.

35. Shanker, R. (2015). Shanker distribution and its applications. International journal of statistics and Applications, 5, 338-348.

36. Smith, R. L. and Naylor, J. C. (1987). A comparison of maximum likelihood and Bayesian estimators for the three-parameter Weibull distribution. Applied Statistics, 36, 358-369.

37. Tejeda, H. A. and Goodwin, B. K. (2008). Modeling crop prices through a Burr distribution and analysis of the correlation between crop prices and yields using a copula method. Paper presented at the annual meeting of the Agricultural and Applied Economics Association, Orlando, FL, USA.

38. Usman, R. M. and Haq, M. A. U. (2020). The Marshall-Olkin extended inverted Kumaraswamy distribution: Theory and applications. Journal of King Saud University-Science, 32(1), 356-365.

39. Wingo, D. R. (1993). Maximum likelihood methods for fitting the Burr type XII distribution to multiply (progressively) censored life test data. Metrika, 40, 203-210. 